\title{
CRISPR/Cas9-induced shank3b mutant zebrafish display autism-like behaviors
}

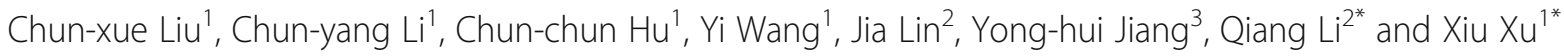

\begin{abstract}
Background: Human genetic and genomic studies have supported a strong causal role of SHANK3 deficiency in autism spectrum disorder (ASD). However, the molecular mechanism underlying SHANK3 deficiency resulting in ASD is not fully understood. Recently, the zebrafish has become an attractive organism to model ASD because of its high efficiency of genetic manipulation and robust behavioral phenotypes. The orthologous gene to human SHANK3 is duplicated in the zebrafish genome and has two homologs, shank3a and shank3b. Previous studies have reported shank3 morphants in zebrafish using the morpholino method. Here, we report the generation and characterization of shank3b mutant zebrafish in larval and adult stages using the CRISPR/Cas9 genome editing technique.

Methods: CRISPR/Cas9 was applied to generate a shank3b loss-of-function mutation (shank36 ${ }^{-1}$ ) in zebrafish. A series of morphological measurements, behavioral tests, and molecular analyses were performed to systematically characterize the behavioral and molecular changes in shank3b mutant zebrafish.

Results: shank36 ${ }^{-1-}$ zebrafish exhibited abnormal morphology in early development. They showed reduced locomotor activity both as larvae and adults, reduced social interaction and time spent near conspecifics, and significant repetitive swimming behaviors. Additionally, the levels of both postsynaptic homer1 and presynaptic synaptophysin were significantly reduced in the adult brain of shank3b-deficient zebrafish.

Conclusions: We generated the first inheritable shank3b mutant zebrafish model using CRISPR/Cas9 gene editing approach. shank $3 b^{-/-}$zebrafish displayed robust autism-like behaviors and altered levels of the synaptic proteins homer1 and synaptophysin. The versatility of zebrafish as a model for studying neurodevelopment and conducting drug screening will likely have a significant contribution to future studies of human SHANK3 function and ASD.
\end{abstract}

Keywords: shank3, CRISPR/Cas9, Zebrafish, ASD, Social behavior, Animal model

\section{Background}

SHANK3 is a master scaffolding protein enriched at the postsynaptic density of excitatory glutamatergic synapses in the brain that has critical roles in synaptogenesis and synaptic function [1-6]. SHANK3 is the key gene implicated in the neurobehavioral features of individuals with chromosome 22q13.3 deletion syndrome or Phelan-McDermid syndrome (PMS) [7, 8]. Moreover, genetic studies have identified point mutations in the SHANK3 gene in cases of autism spectrum disorder

\footnotetext{
* Correspondence: liq@fudan.edu.cn; xuxiu@shmu.edu.cn

${ }^{2}$ Center for Translational Medicine, Institute of Pediatrics, Shanghai Key Laboratory of Birth Defect, Children's Hospital of Fudan University, 399 Wanyuan Road, Shanghai 201102, China

'Division of Child Health Care, Children's Hospital of Fudan University, 399

Wanyuan Road, Shanghai 201102, China

Full list of author information is available at the end of the article
}

(ASD) that establish the causal role of SHANK3 mutations in $~ 1 \%$ of individuals with ASD [9-11].

Animal models of ASD that mimic SHANK3 genetic detects have facilitated a better understanding of the underlying molecular mechanisms and development of more effective treatments $[2,12]$. More than a dozen different lines of Shank 3 mutant mice have been generated and characterized [4, 13-15]. Almost all Shank3 mutant mice exhibit some of the core behavioral features of ASD $[4,13,14]$. Despite significant advantages, there are clear disadvantages associated with the use of rodent models. For example, it remains difficult to scale up for high-throughput drug screening in rodent models [12]. Compared to rodent models, zebrafish (Danio rerio) exhibit much more efficient reproduction, rapid external development [12, 16, 17], and optical transparency [17]. 
Previous studies have shown that the gene orthologous to human SHANK3 is duplicated in zebrafish as shank3a (in chromosome 18) and shank3b (in chromosome 4) $[18,19]$. Transient knockdown of both shank3a and shank3b expressions by morpholino method has been reported [19, 20]. However, previously, the analysis of developmental and behavioral characteristics was only conducted within 5 days of post-fertilization (dpf), an early stage of development [19]. In the present study, we generated and characterized the first CRISPR/Cas9 engineered shank3b loss-of-function mutation that is stably transmitted in zebrafish. This model will enable a comprehensive study of a mechanistic link between shank3 loss-of-function and ASD and provide a new experimental platform for high throughput drug screening in the future.

\section{Methods}

\section{Generation of shank3b mutant zebrafish}

The detailed procedure for CRISPR/Cas9 editing in zebrafish was described previously [21, 22]. The shank3b target in this study was $5^{\prime}$-GGGCGTGTTGTTGCCAC GGCCGG-3' (Additional file 1: Table S1). Injection mixtures included $500 \mathrm{pg}$ of Cas 9 mRNA and $120 \mathrm{pg}$ of gRNA. Eighty zebrafish were screened to identify a founder, and the germline mutation frequency was approximately 35\%. Mutant sites were verified by comparison to the WT unaffected sequences (chimerism). Chimeric zebrafish were mated onto a Tu background for three generations to obtain $\operatorname{shank} 3 b^{+/-}$zebrafish. We crossed shank $3 b^{+/-}$males and shank $3 b^{+/-}$females to obtain shank $3 b^{+/+}$, shank $3 b^{+/-}$, and shank $3 b^{-/-}$littermates for all experiments of phenotypic analyses.

\section{Tg (HuC: RFP) transgenic line and zebrafish maintenance} The wild-type (WT) Tu zebrafish strain was acquired from the Institute of Zebrafish, Children's Hospital of Fudan University. The zebrafish were raised and maintained in a standard laboratory environment $\left(28.5^{\circ} \mathrm{C}\right)$ and a $14 \mathrm{~h}$ light $/ 10 \mathrm{~h}$ dark cycle according to a standard protocol $[17,23]$. The $\mathrm{Tg}\left(\operatorname{shank} 3 b^{+/+}-\mathrm{HuC}\right.$ : $\left.\mathrm{RFP}^{+/-}\right)$transgenic line, kindly provided by Dr. Xu Wang (Fudan University), was made via plasmid injection with tol 2 mRNA at singlecell stage followed by screening for germline transmission. The vector was generated by inserting the $\mathrm{HuC}$ promoter [24] upstream of RFP cDNA followed by polyA sequence in a Tol2 destination vector, using multisite Gateway cloning [25]. In order to collect enough eggs efficiently for the RFP imaging experiments, we crossed $T g\left(\operatorname{shank} 3 b^{+/-}-H u C\right.$ :

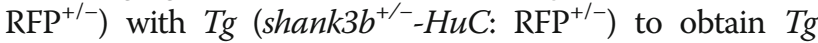
$\left(\operatorname{shank} 3 b^{-/-}-\mathrm{HuC}\right.$ : $\left.\mathrm{RFP}^{+/+}\right)$for the experimental group. We crossed $T g\left(\operatorname{shank} 3 b^{+/+}-\mathrm{HuC}: \mathrm{RFP}^{+/-}\right)$and $T g$

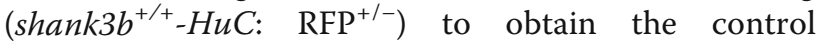

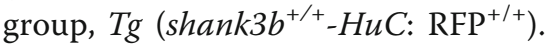

\section{RT-qPCR}

Real-time quantitative polymerase chain reaction (RT-qPCR) was performed in triplicate, with 4-10 zebrafish per sample. Total RNA was extracted from the larval or adult brains using TRIzol reagent (Ambion, USA). Reverse transcription was performed with a PrimeScript $^{\mathrm{TM}}$ RT Reagent Kit (RR037A, TaKaRa, Japan), according to the manufacturer's protocol. Oligo dT primer $(25 \mathrm{pmol})$ and random 6 mers $(50 \mathrm{pmol})$ were added in $10 \mu \mathrm{l}$ mixture to efficiently obtain full-length cDNA. RT-qPCR was performed using a LightCycler 480 apparatus (Roche, Germany) and SuperRealPreMix Plus (Tiangen, China), according to the manufacturers' instructions. Finally, we used the delta delta CT method to calculate the expression levels. The primers used in this study are described in Table S1 in Additional file 1.

\section{Larval activity and light/dark tests}

A ViewPoint setup combined with an automated computer recording system equipped with VideoTrack software was used to measure locomotor activity. The camera was a Point Grey black-and-white camera with a resolution of $1024 \times 768$. Videos were recorded for $60 \mathrm{~min}$ at $25 \mathrm{fps}$ and were pooled into 1-min time bins. The detection threshold was set to 25. Activity was quantified using Zebralab software. The distance traveled by the larvae in the well was measured to analyze general locomotor activity. For all behavioral analyses, we used a commercial Viewpoint tracking system and custom software written in $\mathrm{C}++$. All behavioral assays were analyzed by experimenters who were blinded to the genotypes. To further analyze the variances of different activity intensity scales among WT, shank $3 b^{+/-}$, and shank $3 b^{-/-}$zebrafish, we divided the activity equally into five levels $(10,20,30,40$, and 50) (Additional file 1: Figure S6). Next, we calculated the activity frequency of different activity intensity scales.

Larvae were habituated in 48-well plates, with one animal per well, in our behavioral assessment room, and videos were recorded for $60 \mathrm{~min}$. The diameter of each well was $1.2 \mathrm{~cm}$. After $30 \mathrm{~min}$ of habituation, each larva was recorded for a total of $30 \mathrm{~min}$ with three light/dark cycles (each consisting of $5 \mathrm{~min}$ of light and $5 \mathrm{~min}$ of dark). The light intensity for photo motor response (PMR) was $100 \mathrm{~lx}$ and the frame rate was 25/s.

\section{Open-field test}

Behavioral experiments were conducted between 10 a.m. and 4 p.m. Each tank was $30 \times 30 \times 30 \mathrm{~cm}$, with walls made of opaque partitions, and a video camera was suspended above the tank. Adult male zebrafish were allowed to freely swim inside the tank, and videos were recorded for $30 \mathrm{~min}$. The timing of all supplementary videos began at approximately the 10th $\mathrm{min}$. 
The thigmotaxis test was performed in the tank divided into two equal zones, a peripheral and a central zone. Adult zebrafish swam freely in the tank. The longer the zebrafish stayed in the peripheral zone, the greater their awareness of danger [12]. The time ratio was the time the zebrafish spent in the peripheral zone divided by the total time spent in tank, and the distance ratio was the distance the zebrafish traveled in the peripheral zone divided by the total distance traveled.

\section{Shoaling test}

Adult male zebrafish were acclimated to the novel tank apparatus for 1-2 min before the test [26]. Videos were recorded for $30 \mathrm{~min}$. The shoaling assessment was performed by measuring the inter-fish distance that represents the average of all distance between each zebrafish in a shoal $[27,28]$.

\section{Social preference test}

Social preference testing was performed in a standard mating tank (inner dimensions $21 \times 10 \times 7.5 \mathrm{~cm}$ ). The tank was separated into two halves by a Plexiglas transparent barrier that allowed the zebrafish to swim freely and was provided sufficient visual information to allow the zebrafish to form a social preference. Behavioral recordings typically started after an acclimation period (1-2 min), when zebrafish usually explored the tank. Videos were recorded for $30 \mathrm{~min}$. The zebrafish behaviors were quantified as a distance distribution or as presence in a zone adjacent to the group or conspecifics. The time ratio was the time spent in the conspecific sector divided by the total time. The distance ratio was the distance traveled in the conspecific sector divided by the total distance traveled. The zebrafish tested were all adult males.

\section{Kin preference test}

The specifications of the mating cylinder were the same as those in the social preference test. Two opaque separators divided the cylinder into three compartments. Videos were recorded for $30 \mathrm{~min}$. Kin preference was represented by the ratio of time spent in the kin sector divided by the total time. The zebrafish tested were all adult males.

\section{Western blot and antibodies}

WT and shank3b-/- zebrafish brains were prepared for western blotting by dissociating the tissues in lysis buffer (RIPA, Beyotime Biotechnology, China) and 1\% protease inhibitor mixture Set I (Calbiochem, San Diego, CA, USA). The lysates were then centrifuged at 12,000 rpm for $5 \mathrm{~min}$, and the supernatant was collected and denatured. $20 \mu \mathrm{g}$ of total protein were separated on an SDS-PAGE gel (12\%) and were blotted onto a polyvinylidene difluoride membrane (Bio-Rad Laboratories,
Hercules, CA, USA). Next, the membrane was blocked with $5 \%$ bovine serum albumin for $1-2 \mathrm{~h}$ at room temperature and was incubated with primary antibodies overnight at $4{ }^{\circ} \mathrm{C}$. The membrane was rinsed and incubated with HRP-conjugated secondary antibodies for $2 \mathrm{~h}$. Finally, chemiluminescent detection was performed with an ECL kit (Rockford, IL, USA). ImageJ software was used for the densitometric analysis $(N=3$ for each group).

The synaptophysin (1:2000; ab32594) and homer1 (1:1000; ARP40181_P050) antibodies were purchased from Abcam (Cambridge, UK) and Aviva Systems Biology (San Diego, USA), respectively. The $\beta$-actin antibody was obtained from Biotech Well (1:2000; code No. WB0196, Shanghai, China).

\section{Statistical analysis}

Statistical analyses were performed using GraphPad Prism software. Simple comparisons between adult shank $3 b^{+/+}$ and shank $3 b^{-1-}$ zebrafish were performed with two-sided unpaired Student's $t$ tests. Analysis of variance (ANOVA) tests were used to compare three genotypes. All the experiments were conducted in triplicate using different samples. $P$ values $<0.05$ were considered as statistically significant. Values are presented as mean \pm SEM.

\section{Results}

Conservation of human SHANK family genes in zebrafish Previous analyses have suggested that the zebrafish ortholog of human SHANK3 is duplicated in the zebrafish genome because of the presence of two highly similar copies of human SHANK3: shank3a and shank3b [19]. To further analyze the evolutionary conservation between human and zebrafish, we performed a phylogenetic analysis of the SHANK gene family (SHANK1, SHANK2, and SHANK3). As shown in Additional file 1: Table S2 and Figure S1, SHANK1 and SHANK2 each have only one homolog that is believed to be an ortholog in the zebrafish genome. Consistent with previous reports [18, 19], we identified two homologs, shank3a (1933 aa) and shank3b (1643 aa), in the zebrafish genome. shank3a and shank3b share 59 and 55\% identity with human SHANK3, respectively (Additional file 1: Table S3 and Figure S2; https://blast.ncbi.nlm.nih.gov/ Blast.cgi). shank3a displayed an overall 59\% identity and $68 \%$ similarity with shank3b but close to $100 \%$ identity in several blocks of amino acids within the protein (Additional file 1: Table S4 and Figure S3). This observation supports that shank3a and shank3b may have evolved from the same ancestral DNA during their evolution. Although human SHANK3 was slightly more conserved in shank3a than shank3b, both of them may be relevant to understand the functions of human SHANK3 protein. 


\section{Generation of shank $3 b^{-/-}$zebrafish}

Zebrafish shank3b specific guide-RNA (gRNA) comprising a 23-base sequence was designed for the genespecific editing of exon 2 of shank $3 b$. We generated a shank $3 b$ mutant by co-injection of Cas9 mRNA and gRNA into zebrafish embryos (one-cell stage). DNA sequencing of target-specific PCR products confirmed that the shank $3 b$ targeted allele carried a deletion of 5 bases and an insertion of 13 bases, resulting in a frameshift mutation and truncated protein 90 amino acids after the mutation. The mutation disrupted all known functional domains of the shank3b protein (Fig. 1a; Additional file 1: Figure S4). Homozygous mutants for shank3b (shank3b ${ }^{-/-}$) were obtained from the heterozygotes cross (shank $3 b^{+/-} \hat{0} \times \operatorname{shank} 3 b^{+/-}$) $)$after mating mutants with the original Tu strain for three generations $\left(\operatorname{shank} 3 b^{+/-}\right)$. RT-qPCR analysis confirmed that the expression of Shank3b mRNA was significantly reduced in shank $3 b^{-/-}$ zebrafish (Fig. 1b), whereas the expression of shank3a mRNA was not affected (Fig. 1c). Thus, these results indicated that we have successfully generated a transgenic line of shank3b-deficient zebrafish.

\section{Morphological analysis of shank $3 b^{-/-}$zebrafish}

We measured morphological changes in shank $3 b^{-/-}$zebrafish to examine the consequences of shank $3 b$ deficiency during zebrafish development. Compared with shank $3 b^{+/+}$and shank $3 b^{+/-}$zebrafish, a significantly greater proportion of shank $3 b^{-/-}$zebrafish died (shank $3 b^{+/+}, 3 \%$; shank $3 b^{+/-}, 9 \%$; shank $\left.3 b^{-/-}, 20 \%\right)$ and exhibited morphological changes at a very early stage (1 dpf). The morphological changes included neurodevelopmental delay, tail bending, and a reduction of melanin content in eye (Fig. 2a, b). However, over the course of development, these differences in the general

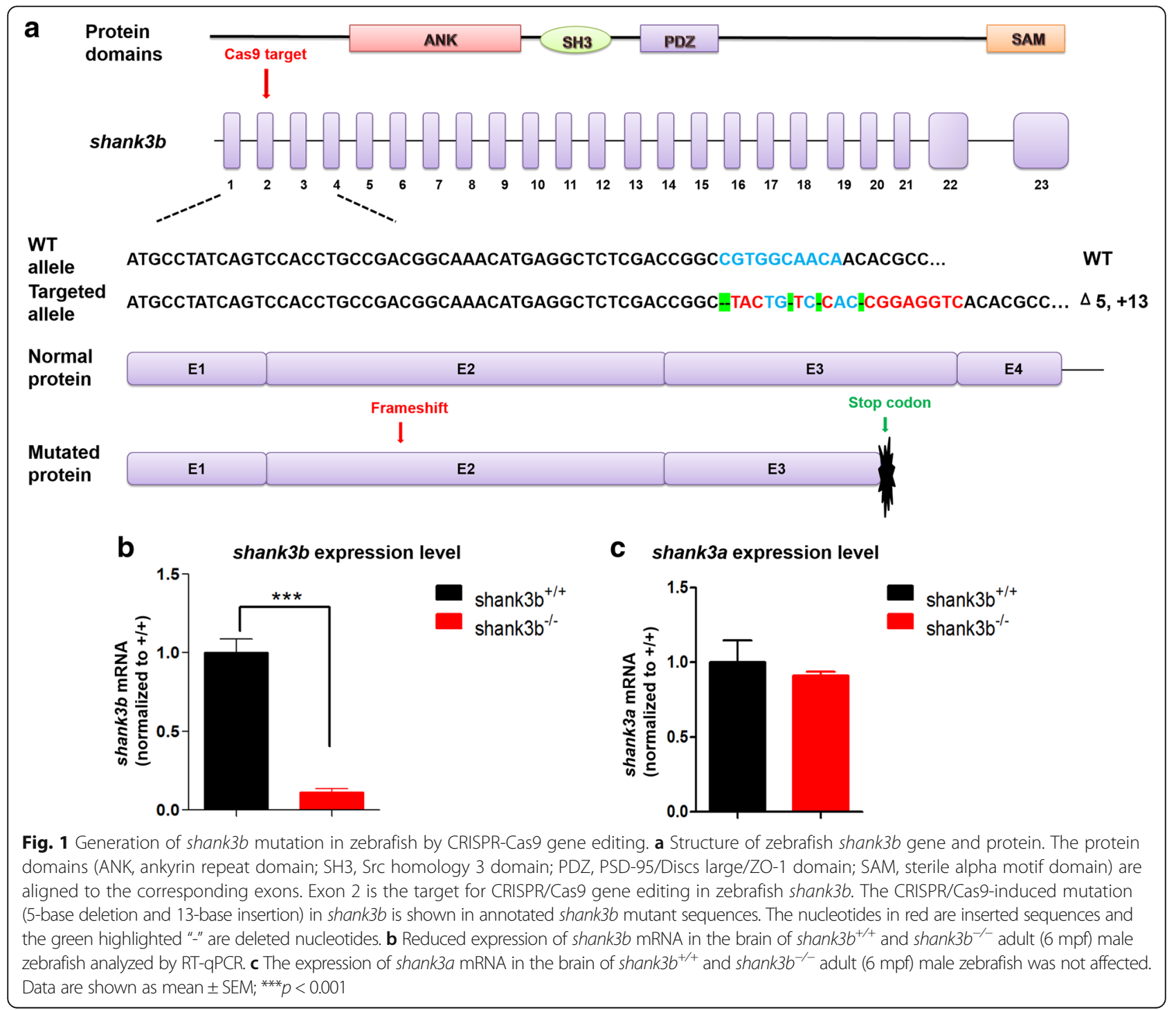




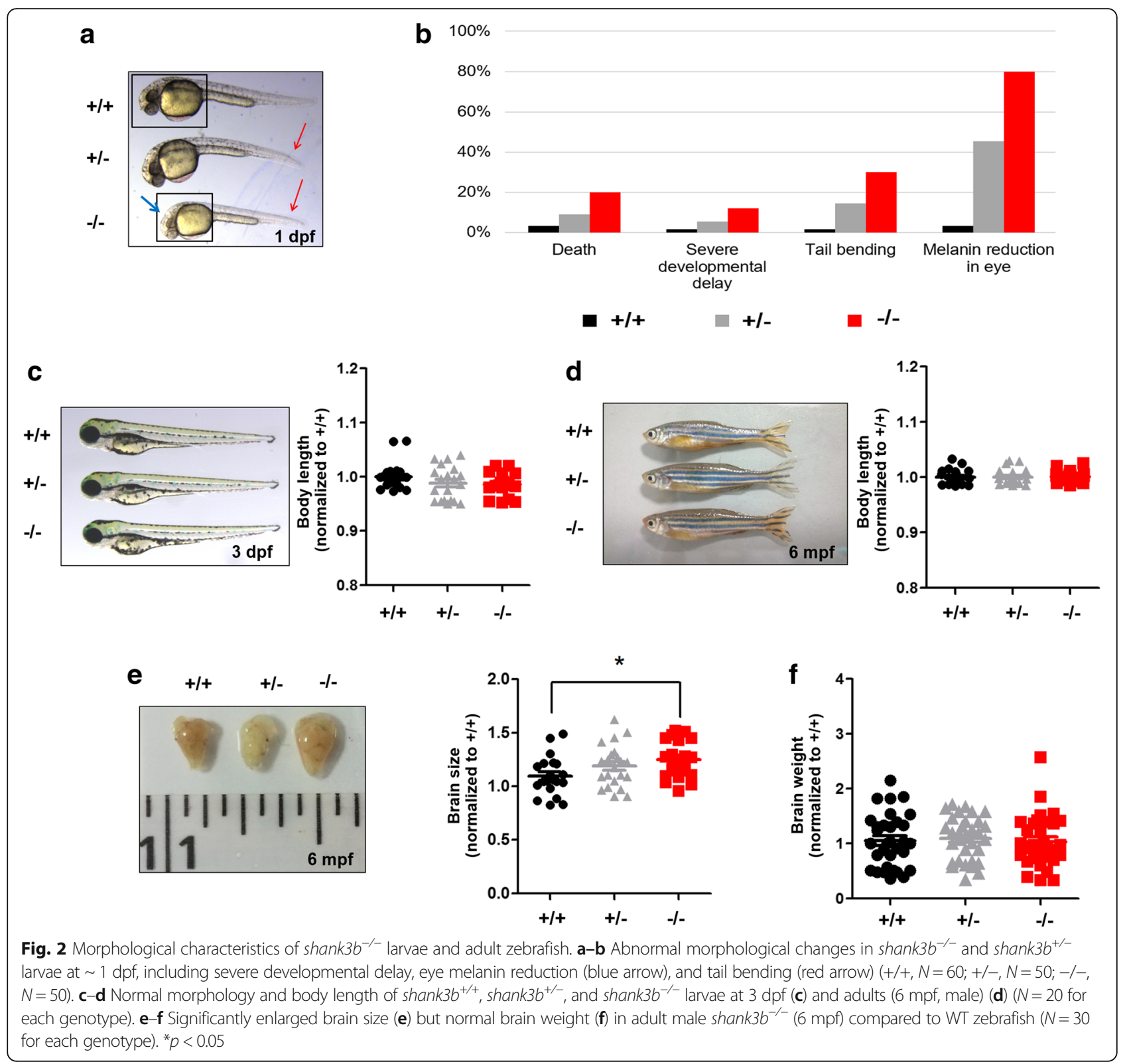

phenotypes gradually become less noticeable (Fig. 2c, d). To determine whether there is a maternal or paternal origin effect on the phenotypes observed among shank $3 b^{-/-}$zebrafish, shank $3 b^{-/-}$females were crossed with WT males and shank $3 b^{-/-}$males were crossed with WT females, respectively. We compared the morphological phenotypes of the offspring from these two breeding schemes and did not find any significant differences (Additional file 1: Figure S5A).

The brain size of adult shank $3 b^{-/-}$zebrafish was significantly larger than that of shank $3 b^{+/+}$zebrafish $\left(p=0.01\right.$, Fig. 2e), whereas the weight of shank $3 b^{-/-}$ brains was comparable to that of $\operatorname{shank} 3 b^{+/+}$and shank $3 b^{+/-}$brains (Fig. 2f). shank $3 b^{-/-}$larvae exhibited impaired locomotor activity To determine whether the loss of function of shank $3 b$ modulates the larval behaviors during development, the frequency was measured at five activity intensities $(10$, $20,30,40$, and 50) among shank3b $b^{+/+}$, shank $3 b^{+/-}$, and shank $3 b^{-/-}$zebrafish (Additional file 1: Figure S6). The spontaneous activity of individual larva was measured for $30 \mathrm{~min}$ in a 48 -well plate at 2, 5, and $7 \mathrm{dpf}$ under light exposure (full light strength is $100 \mathrm{~lx}$ ). Compared with shank $3 b^{+/+}$larvae, shank $3 b^{-/-}$and shank $3 b^{+/-}$ larvae exhibited a trend of reduced activity at $2 \mathrm{dpf}$, but the differences did not reach statistically significance (Fig. 3a). shank $3 b^{-/-}$and shank $3 b^{+/-}$larvae moved significantly less than shank $3 b^{+/+}$larvae at higher activity 

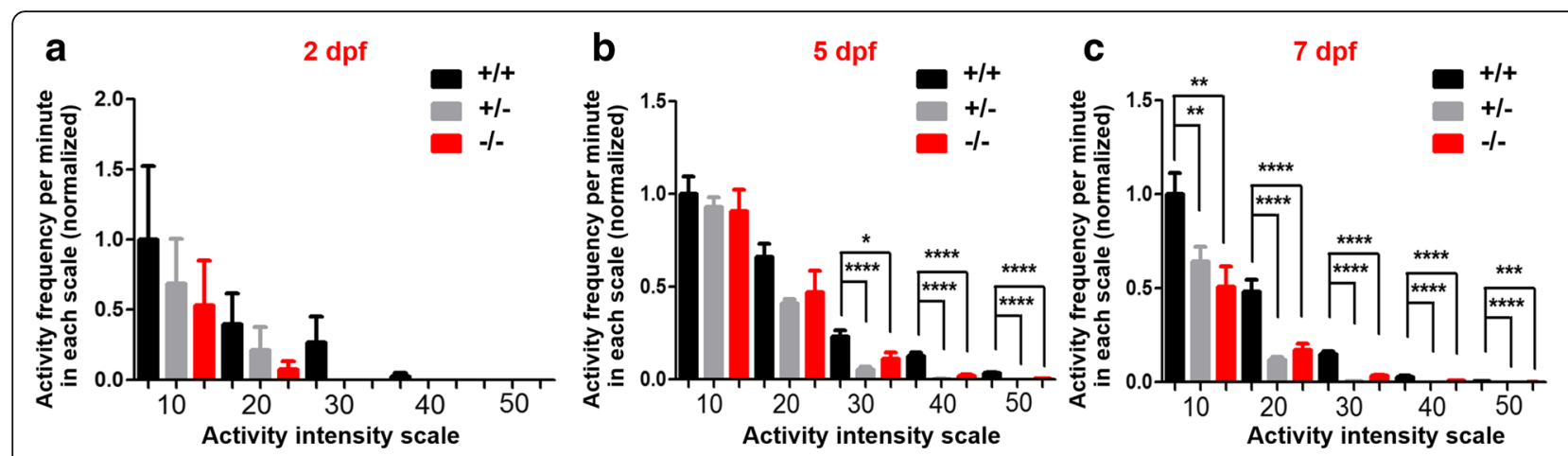

d

Light-dark
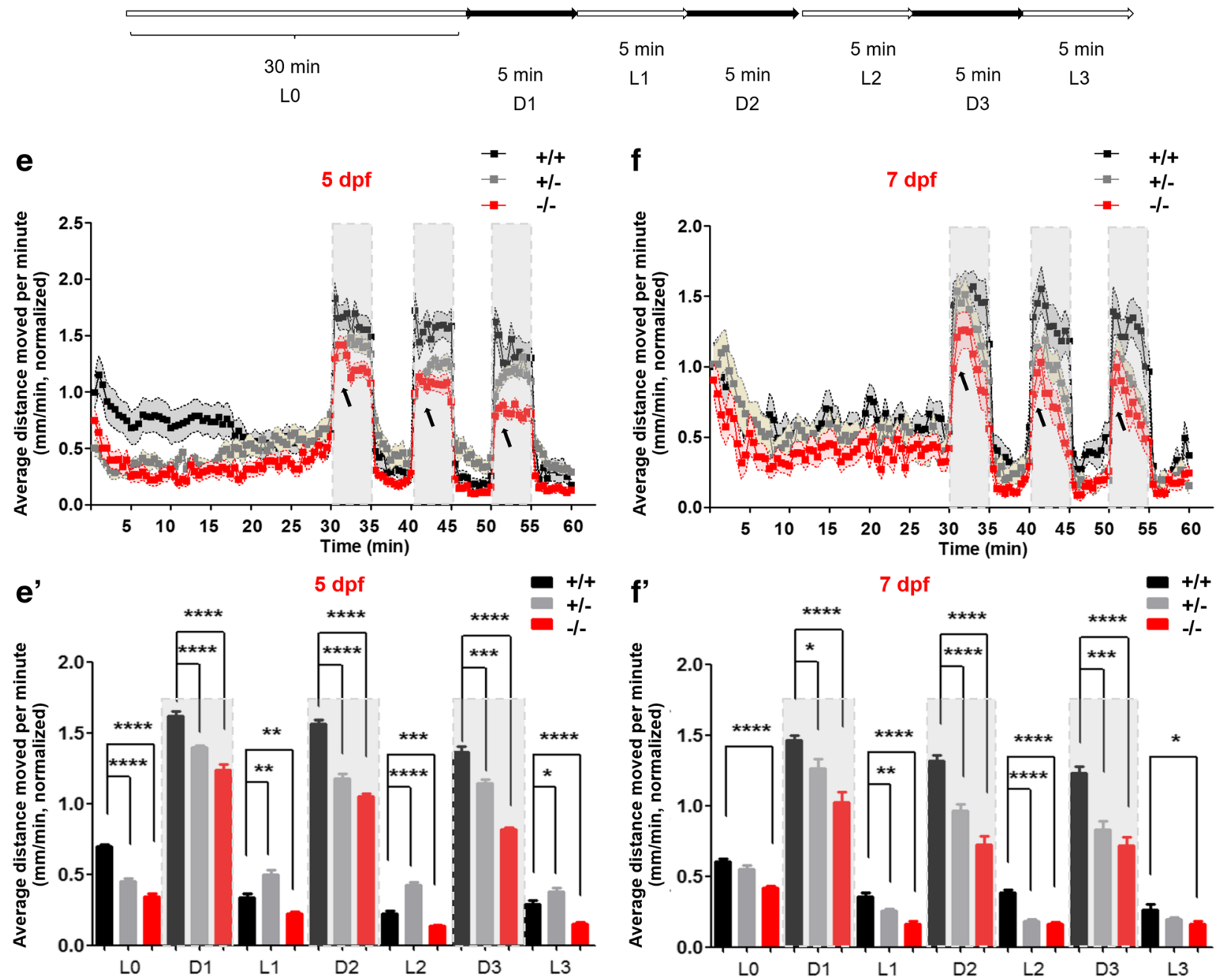

Fig. 3 shank $3 b^{-/-}$larvae displayed impaired locomotion activity. a-c Spontaneous activity of shank $3 b^{+/+}$, shank $3 b^{+/-}$, and shank $3 b^{-/-}$larvae was significantly reduced at 5 and $7 \mathrm{dpf}$, but not at $2 \mathrm{dpf}$. The $X$ axis shows the intensity scale of the activity and $Y$ axis shows the normalized activity frequency traveled by larvae in 1-min bin on each intensity scale ( $N=24$ for each genotype). $\mathbf{d}-\mathbf{f}^{\prime}$ Light/dark test of shank $3 b^{+/+}$, shank $3 b^{+/-}$, and shank $3 b^{-1-}$ larvae at 5 and $7 \mathrm{dpf}$. The activity was recorded during 30 min of light (LO) and three 5 -min light/dark intervals (D1/L1, D2/L2, and D3/L3) (d). The average distance moved within each 1-min bin under either light or dark conditions is plotted. Experiments were performed at 5 $\mathrm{dpf}$ (e and $\mathbf{e}^{\prime}$ ) and $7 \mathrm{dpf}\left(\mathbf{f}\right.$ and $\mathbf{f}^{\prime}$ ). The vertical axis shows the normalized distance (millimeters) traveled by larvae in each 1-min bin. Data are shown as mean \pm SEM $\left(N=24\right.$ for each genotype); ${ }^{*} p<0.05$, ${ }^{* *} p<0.01,{ }^{* * *} p<0.001$, ${ }^{* * * *} p<0.0001$ 
scales on $5 \mathrm{dpf}$ (Fig. 3b), and at all activity scales on 7 dpf (Fig. 3c).

We also examined the responses evoked by light changes (light/dark switch, $100 \mathrm{~lx}$ for brightness and $0 \mathrm{~lx}$ for dark). After a 30-min habituation period, each larva displayed relatively stable activity and was recorded for $30 \mathrm{~min}$ over three light/dark cycles (each consisting of $5 \mathrm{~min}$ in light and $5 \mathrm{~min}$ in dark setting per cycle, Fig. 3d). Under continuous illumination, the total distance traveled was measured. Compared with shank $3 b^{+/+}$larvae, shank $3 b^{-/-}$and shank $3 b^{+/-}$larvae traveled significantly less, and shank $3 b^{-/-}$larvae performed significantly worse than $\operatorname{shank} 3 b^{+/-}$larvae. Light-to-dark transitions elicited sudden increases of total distance traveled, while dark-tolight transitions resulted in sudden decreased distance traveled (Fig. 3e, f, e', f'). However, shank $3 b^{-/-}$and shank $3 b^{+/-}$larvae showed fewer responses to changes in illumination (arrows in Fig. 3e, f).

To test whether there is a maternal or paternal origin effect on behavioral phenotypes, we compared larval activity and light/dark switch responses in the offspring of shank $3 b^{-/-}$female and shank $3 b^{-/-}$male zebrafish. However, no significant differences were observed among these two groups (Additional file 1: Figs. S5B-5F and S5B'-5F').

\section{shank $3 b^{-/-}$adult zebrafish displayed impaired locomotor} activity and abnormal repetitive movements

The locomotor activity of adult shan $k 3 b^{-/-}$zebrafish was also examined in an illuminated tank (Fig. 4a). Significantly reduced swimming velocity was observed in shank $3 b^{-/-}$zebrafish, compared with shank $3 b^{+/+}$zebrafish (Fig. 4b). Although shank $3 b^{+/+}$zebrafish displayed reduced velocities with increased time in the tank, shank $3 b^{-/-}$zebrafish showed steadily lower locomotor activity throughout the examination window (Fig. 4c).

To determine whether disruption of shank $3 b$ alters thigmotaxis, the two groups of adult zebrafish were assessed for the percentage of time spent and the distance traveled in the center vs. the peripheral zones in a new water tank (Fig. 4a). Compared with shank $3 b^{+/+}$ zebrafish, shank $3 b^{-/-}$zebrafish spent considerably more time and traveled longer distances in the center of the tank than in the peripheral area (Fig. 4d, e).

When the trajectories of activity and pattern of swimming were analyzed in a blinded fashion, we noticed that shank $3 b^{-/-}$zebrafish exhibited a significantly higher frequency of stereotypical behaviors (Fig. 4f, g; Additional file 1: Table S5) than shank $3 b^{+/+}$zebrafish (Additional file 2: Movie S1). The repetitive behaviors include repetitive or stereotypic figure "8" swimming, circling, cornering, and walling (Additional file 3: Movie S2, Additional file 4: Movie S3, Additional file 5: Movie S4, Additional file 6: Movie S5).

\section{shank $3 b^{-/-}$zebrafish displayed impaired social preference} behaviors

It is known that wild-type zebrafish typically swim together in a school that reflects the social nature of the species. We therefore used the shoaling test to assess the social cohesion among homogeneous groups of zebrafish $[26,29]$. In this assay, adult shank $3 b^{+/+}$or shank $3 b^{-/-}$ zebrafish were placed in the testing tank. The average inter-fish distance was measured every $30 \mathrm{~s}$ for all pair combinations (Fig. 5a). As shown in Fig. 5b, shank $3 b^{+/+}$ zebrafish typically swim as schools, which is characterized by a short inter-fish distance, a short average diameter of the group, and a clear polarization (Additional file 7: Movie S6), whereas shank3b $b^{-/}$zebrafish exhibited larger and looser schools, increased average inter-fish distance, and a greater number of zebrafish swimming away from the group and spending more time outside the group (Additional file 8: Movie S7).

The social preference and interaction tests were subsequently performed using a two-sector tank, divided in the middle with clear Plexiglas to allow visualization. A group of six conspecific zebrafish was placed in the right side, and a single shan $k 3 b^{+/+}$or shank $3 b^{-/-}$test zebrafish was placed on the left side (Fig. 5c). shank $3 b^{+/+}$zebrafish generally contacted the group on the right side and spent more time in the conspecific sector rather than the empty sector, showing a strong group tendency (Fig. 5d; Additional file 9: Movie S8). In contrast, shan $k 3 b^{-/-}$zebrafish spent their time evenly throughout the region and exhibited reduced duration and frequency of social contacts with the peer group (Additional file 10: Movie S9). Quantitatively, compared with shank $3 b^{+/+}$ zebrafish, shank $3 b^{-/-}$zebrafish exhibited a significantly decreased time ratio (Fig. 5e) and distance ratio (Fig. 5f) in the conspecific sector.

In the related kin recognition and preference test, the zebrafish $\left(\operatorname{shank} 3 b^{+/+}\right.$or shank $3 b^{-/-}$) was placed in the middle of a three-chamber apparatus with Plexiglas dividers, with kin zebrafish placed on the right and nonkin (red color) zebrafish placed on the left (Fig. 5g). shank $3 b^{+/+}$zebrafish typically spent more time near the kin group (conspecific and same color) than near the non-kin group (Additional file 11: Movie S10), indicating kin recognition and preference. In contrast, shank $3 b^{-/-}$ zebrafish swam in a loose and irregular manner, and the total time spent parallel to conspecifics was much less than that found in shank $3 b^{+/+}$zebrafish (Fig. 5h; Additional file 12: Movie S11).

\section{shank3b deficiency affected neurodevelopment in larvae}

To further study neural development, the $H u C$-RFP transgenic line that is widely expressed in the nervous system during embryonic development was used in 


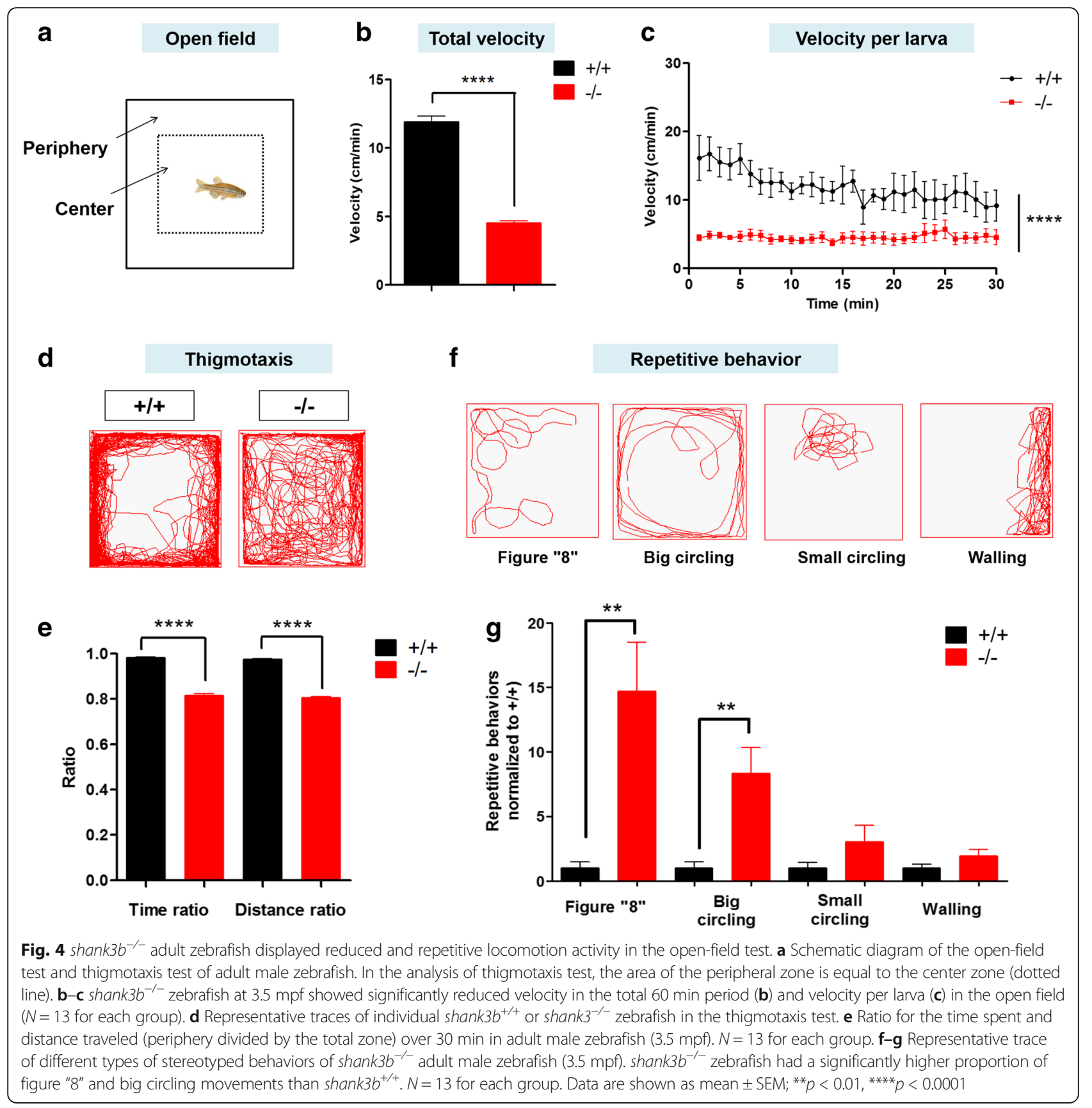

this study. The $H u C$-RFP transgene, in which the $H u C$ promoter drives RFP expression, enables clear and direct visualization of neurodevelopment in transparent larvae (Fig. 6a-c). Compared with shank $3 b^{+/+}$larvae, the expression of the RFP reporter was significantly reduced in shank $3 b^{-/-}$larvae from 1 to $3 \mathrm{dpf}$, indicating that the neurodevelopment of shank $3 b^{-/-}$larvae was altered (Fig. 6a'-c'). In addition, the differences in RFP expression at $1 \mathrm{dpf}$ decreased over time, consistent with the developmental delay shown in Fig. 2. shank $3 b$ deficiency resulted in reduced homer 1 and synaptophysin protein levels in the adult zebrafish brain Shank3 is a core scaffolding protein located at the postsynaptic density [1]. Significantly reduced Homer1, a major postsynaptic protein, is reported in Shank3 mutant mice [4]. We therefore examined homer1 protein levels in adult shank $3 b^{-/-}$zebrafish brains. We found that the level of homer1 protein was significantly decreased $\left(27 \%\right.$ of $\left.\operatorname{shank} 3 b^{+/+}\right)$in the brain of shank $3 b^{-/-}$zebrafish $(n=3$, mean \pm SD, 0.27 

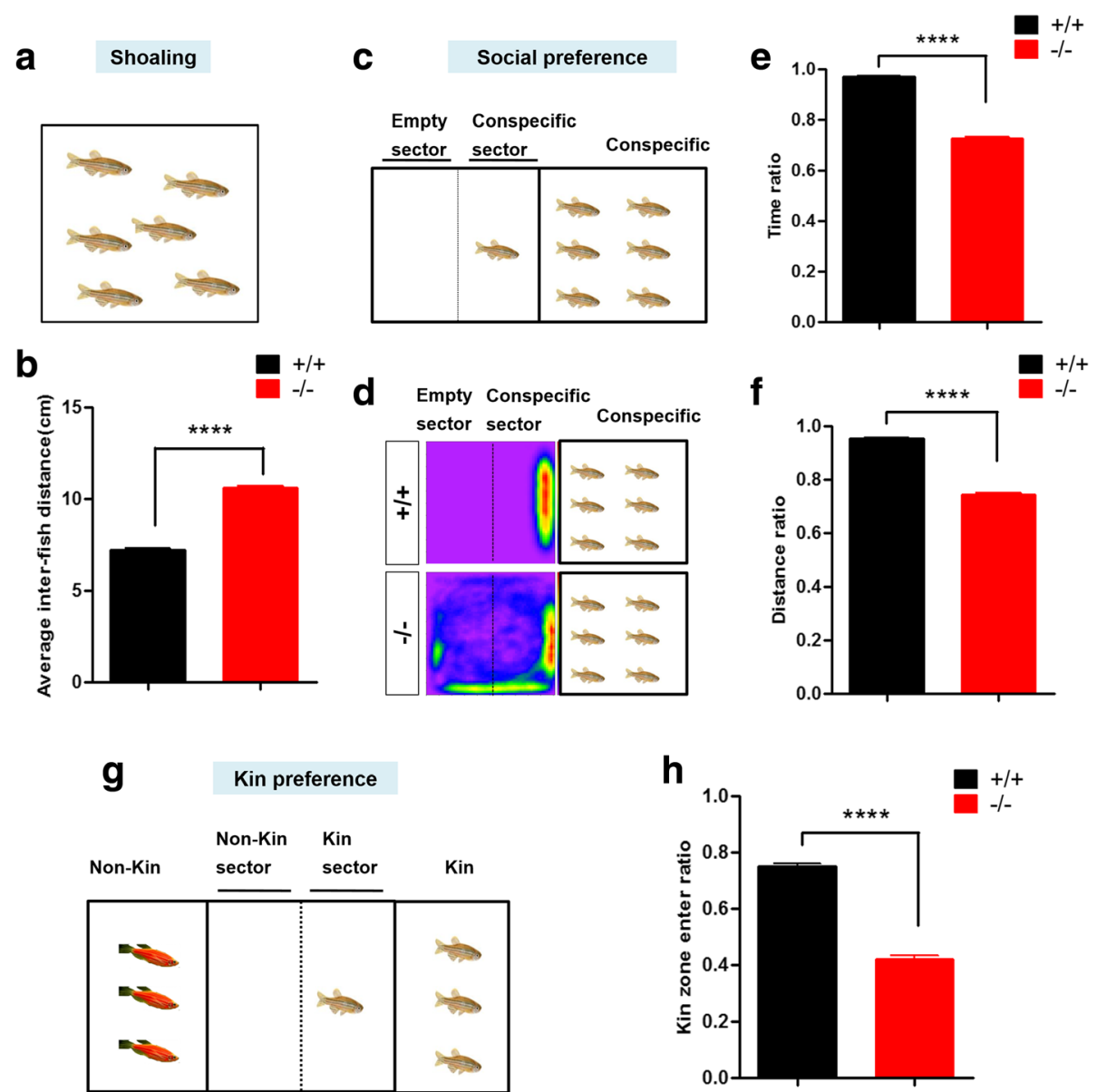

Fig. 5 shank $3 b^{-/-}$zebrafish displayed social interaction defect. a-b Schematic of shoaling test (a) and significantly increased inter-fish distance of adult male shank $3 b^{-1-}$ zebrafish (3.5 mpf) (b). $\mathrm{N}=18$ for each group. c-f Schematic of social preference test of adult male zebrafish (3.5 mpf) (c).

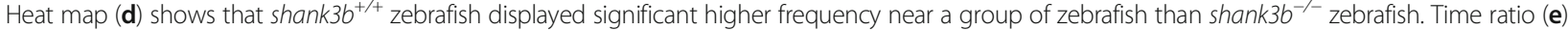
and distance ratio $(\mathbf{f})$ in the conspecific sector were significantly reduced in shank $3 b^{-/-}$zebrafish compared to shank $3 b^{+/+}$zebrafish. $N=16$ for each group. $\mathbf{g}-\mathbf{h}$ Schematic of kin recognition and preference test of adult male zebrafish (3.5 $\mathrm{mpf}$ ( $\mathbf{g}$ ) and significantly reduced ratio of kin zone entering

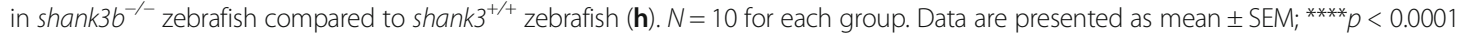

$\pm 0.02)$ compared with $\operatorname{shank} 3 b^{+/+}$zebrafish $(n=3$, $1.00 \pm 0.25$; Fig. 7a).

Shank3 deletion has also been reported to impair synaptic transmission, and neurexin and neuroligin mediated trans-synaptic signaling [30]. We investigated whether presynaptic proteins were also affected in the shank $3 b^{-1-}$ zebrafish brain. Synaptophysin is exclusively located in synaptic vesicles and is generally used as a marker for presynaptic terminals [31]. As shown in Fig. $7 \mathrm{~b}$, the levels of synaptophysin were markedly decreased in shank $3 b^{-/-}$zebrafish (49\% of shank $3 b^{+/+}$; $n=3,0.54 \pm 0.13)$ compared with shank $3 b^{+/+}$zebrafish $(n=3,1.10 \pm 0.31)$.

\section{Discussion}

In this study, we generated the first shank3b loss-offunction mutation in zebrafish using the CRISPR/Cas9 gene editing method and reported the morphological, behavioral and neurological characterizations of shank3b zebrafish mutants at both early developmental stage and adulthood. The shank3b deficiency caused partial lethality during early development as well as defective and delayed neurodevelopment at the larval stage. The brain volume of shank $3 b^{-1-}$ zebrafish is enlarged but the brain weight is comparable to $\operatorname{shank} 3 b^{+/+}$, which may indicate the ventricles in shank $3 b^{-/-}$are larger than in WT zebrafish. This observation is reminiscent of the enlarged ventricular size frequently reported in human PMS patients $[32,33]$. However, it is interesting to note that the defective and delayed neurodevelopment in shank $3 b^{-1-}$ larvae becomes less noticeable later in development. The exact reason for the finding is not immediately clear but may support a different functional role of shank3b protein at different developmental stages.

shank $3 b^{-/-}$zebrafish in adulthood display significantly abnormal behaviors while shank $3 b^{+/-}$zebrafish showed 

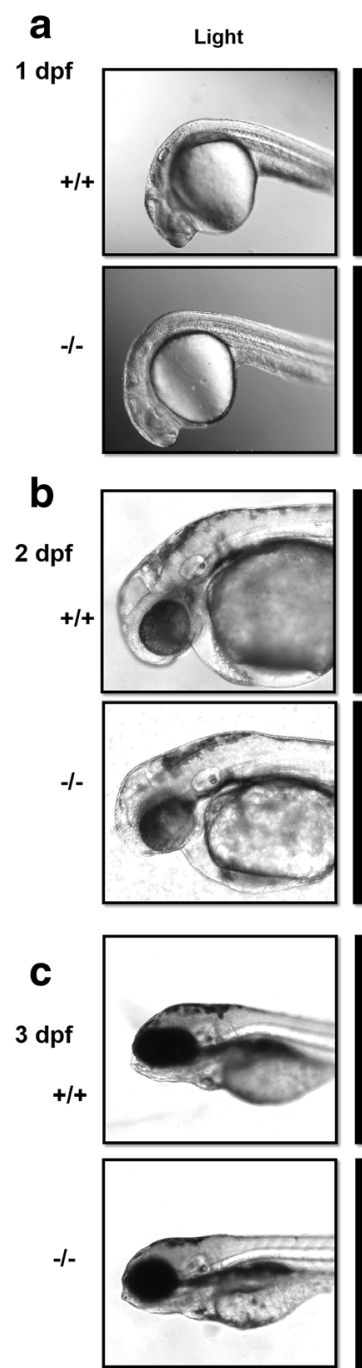
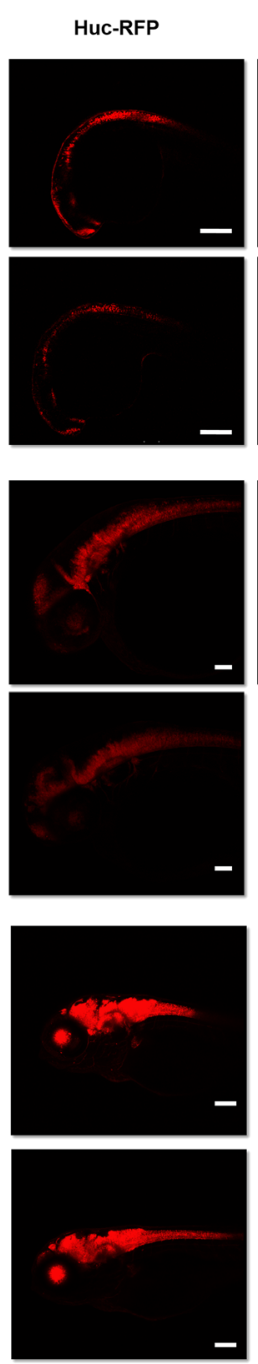
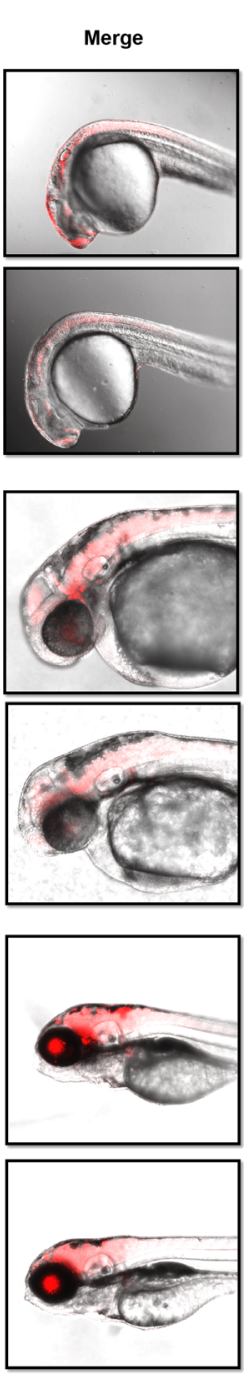

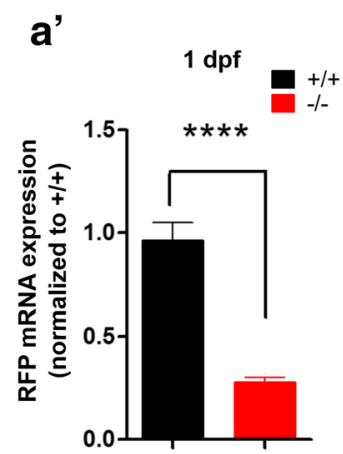

b'
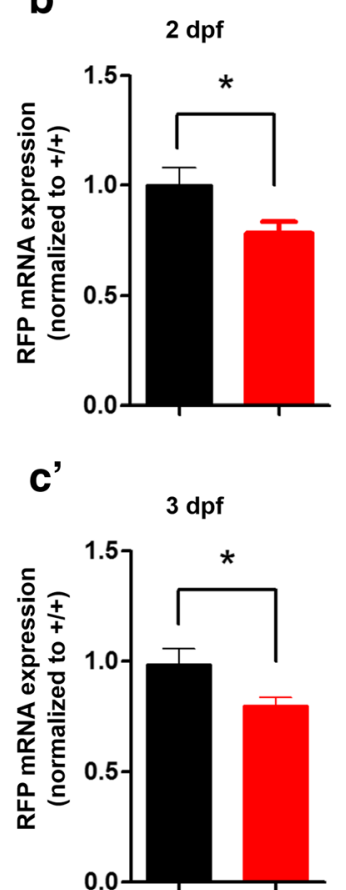

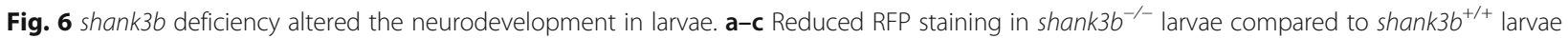
(1 dpf, 2 dpf, and 3 dpf) using Huc: RFP transgene line zebrafish. The difference is the most prominent at 1 dpf. Scale bar, 100 Hm. $\mathbf{a}^{\prime}-\mathbf{c}^{\prime}$ RT-qPCR results of RFP expressions from $(\mathbf{a}-\mathbf{c})$ larvae. $N=8$ for each group. Data are presented as mean \pm SEM; ${ }^{*} p<0.05$, ${ }^{* * * *} p<0.0001$

intermediate phenotypes compared to those of shank3b -/- and shank $3 b^{+/+}$zebrafish. The phenotypes observed in shank3b $b^{+/-}$zebrafish are analogous to the haploinsufficiency of SHANK3 seen in PMS and SHANK3-related disorders $[9,34]$. The observed early-stage developmental defects and abnormal behaviors in both shank $3 b^{+/-}$ and shank $3 b^{-/-}$zebrafish larvae are different from Shank3 rodent models, in which early developmental defects have not been reported, and phenotypes in heterozygous mutants are generally not significant [4, 35, 36]. The reason for these differences between the two species is not clear. Considering that zebrafish have both shank3a and shank3b homologs to human SHANK3, it is somewhat unexpected or counterintuitive that shank3b mutant zebrafish have more prominent phenotypes for survival and behavior. An alternative explanation for the behavioral phenotypes is that the more significant abnormal behaviors in shank $3^{+/-}$zebrafish are because behavioral assays in zebrafish are more sensitive than that in rodents.

The ortholog of human SHANK3 is duplicated in the zebrafish genome as shank3a and shank3b during teleost evolution [12, 17]. The duplicated and conserved shank3a and shank3b share high identity at the amino acid level and are expected to have a similar function in zebrafish [17]. In a previous study, Kozol et al. reported the knock down of shank3a and shank3b by morpholino and observed embryonic defects in both morphants and impaired touch-induced startle responses in shank3a morphants [19]. However, abnormal ASD-like behaviors were not detected due to the limitations of morpholino technology. It would be interesting to compare the 

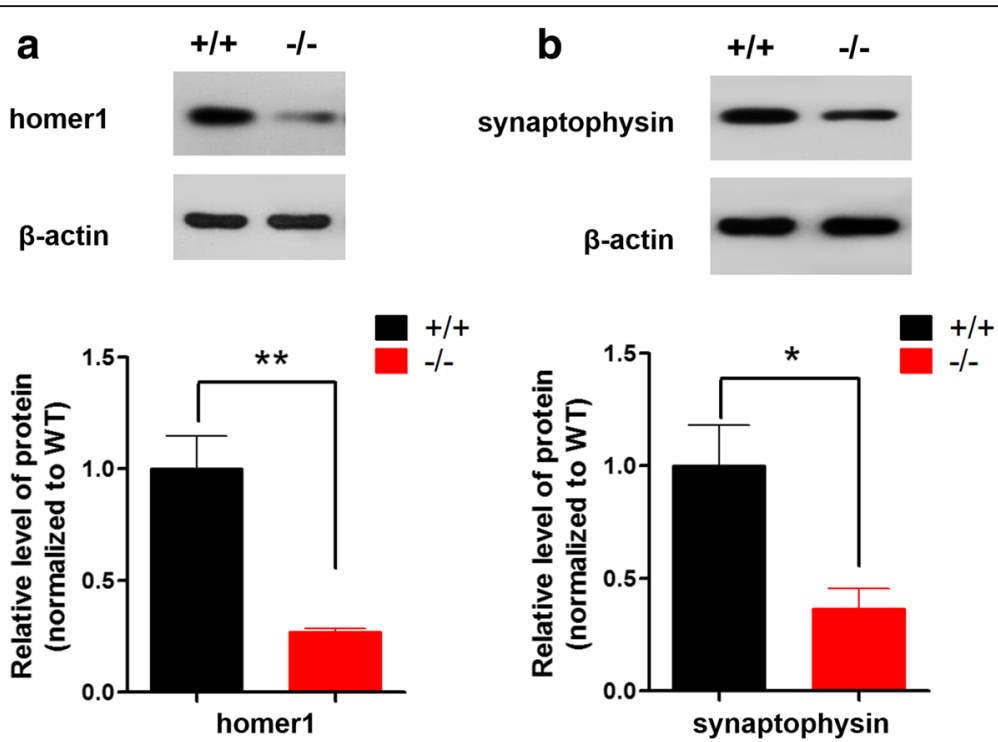

Fig. 7 shank3b deficiency resulted in the reduction of post- and presynaptic proteins in adult zebrafish brain. a Quantitative immunoblot blot analysis showed that the postsynaptic protein homer 1 was significantly decreased $\left(27 \%\right.$ of shank $\left.3 b^{+/+}\right)$in the shank $3 b^{-1-}$ male zebrafish brain relative to shank $3 b^{+/+}$zebrafish ( $3.5 \mathrm{mpf}, \mathrm{N}=3$ for each group). $\mathbf{b}$ The expression of presynaptic synaptophysin protein was markedly reduced

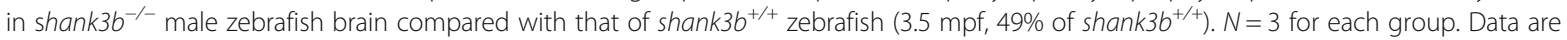
presented as mean \pm SEM; ${ }^{*} p<0.05,{ }^{* *} p<0.01$

phenotypes of shank $3 a$ and $\operatorname{shank} 3 b$ mutants engineered by CRISPR/Cas9 in parallel or even the phenotypes of shank $3 a$ and shank $3 b$ double mutants in the future.

In recent years, the zebrafish has become an attractive alternative model for ASD researchers [19, 27, 37]. Many behavioral assays have been developed in zebrafish models, including the assessment of social interaction, novelty seeking, courtship, inhibitory avoidance, fear and anxiety responses, repetitive/stereotyped behaviors, seizures, and aggression [12, 38-41]. We employed some of the behavioral assays in the analyses of shank $3 \mathrm{~b} \mathrm{mu-}$ tant zebrafish and found striking differences in social and repetitive behavioral domains between shank $3 b^{-1-}$ and shank $3 b^{+/+}$zebrafish. For instance, in shoaling and kin-preference assays, shank $3 b^{-/-}$zebrafish preferred to swim in loose schools and showed significantly decreased preference for conspecifics. These abnormal behaviors are reminiscent of reduced social interaction in the home cage or abnormal social novelty and preference using the three chamber paradigm reported in several lines of Shank3 mutant mice [35, 36, 42, 43]. In the open field, shank $3 b^{-/-}$zebrafish displayed abnormal locomotor activity, such as figure "8" and "circling" movements that are apparently repetitive. Similarly, repetitive behavior measured by increased self-grooming has been observed in several lines of Shank3 mutant mice $[4,42]$. However, like many other behavioral findings observed in animal models, the challenge remains to determine whether the abnormal behaviors observed in shank3b-deficient zebrafish can be directly translated to human SHANK3-related ASD. The study of the predictive validity of these abnormal behaviors to ASD may be warranted in the future, when feasible. Positive results could potentially provide further support for the translational value of these behavioral phenotypes. It also remains to be seen if these assays are universally valid and effective for ASD models caused by different genetic defects. Clinical and molecular heterogeneity have been well recognized in ASD in humans [44]. Additional behavioral assays are certainly needed to assess face validity for ASD-like behaviors, and also for common comorbidities such as seizures and cognitive impairments.

Our finding of reduced postsynaptic homer1 protein levels in shank3b-deficient zebrafish is consistent with the known function of SHANK3 as a scaffolding protein at the postsynaptic density from studies of Shank3 mutant mice $[4,45]$. This finding, although limited, would suggest that the molecular mechanism-associated SHANK3 deficiency may be conserved between different species. It would be interesting to examine if the same defect occurs in shank3a-deficient zebrafish. The finding of significantly reduced synaptophysin protein levels in the brain of shank $3 b^{-1-}$ zebrafish is novel, as synaptophysin is a known presynaptic protein [31]. This observation implies that shank $3 b$ deficiency may affect presynaptic function directly or via a trans-synaptic mechanism in zebrafish. Several recent studies have suggested that SHANK3 protein is located at the presynaptic terminus in the brain as well as in dorsal root ganglion neurons in rodents [46]. Our finding in 
zebrafish also potentially suggests a role of shank3 protein in the presynaptic terminus. Future studies on the presynaptic function of shank $3 b^{-/-}$are warranted and may shed additional insight in this direction.

The amenability to high-throughput drug screening is a tremendous advantage of the zebrafish model. The list of confirmed ASD-causing genes continues to grow, but the development of targeted molecular treatments significantly lags behind. A validated experimental platform that can translate the genetic discoveries to drug screening at a fast pace is urgently needed. We believe that the shank $3 b^{-/-}$model described in this study and other similar ASD zebrafish models will lay an important foundation for the development of a productive drug screening program for ASD and may ultimately lead to the discovery of an effective intervention.

\section{Conclusions}

For the first time, we successfully generated a shank3b -/- zebrafish model that displays robust autism-like behavioral characteristics. Reduced levels of the postsynaptic scaffolding protein homer1 in shank $3 b^{-/}$zebrafish suggest a high conservation of the molecular mechanism underlying SHANK3 deficiency among different species. The reduced levels of synaptophysin in the brain of shank $3 b^{-1-}$ zebrafish also provide further evidence supporting the potential role of shank 3 in presynaptic terminus. The shank $3 b$ mutant zebrafish represents a valuable model to dissect the molecular pathogenesis and conduct high-throughput drug screening for SHANK3-related disorders in the future.

\section{Additional files}

Additional file 1: Table S1. gRNA gene-target sequences, oligonucleotides for PCR knock-out validation, and RT-qPCR probes used in this study. Table S2. SHANK family sequences used in this study. Table S3. Homology analysis of zebrafish shank3a and shank3b compared with human SHANK3. Table S4. Homology comparison between zebrafish shank3a and shank3b. Table S5. Repetitive behaviors of shank $3 b^{-1-}$ adult male zebrafish (3.5 mpf). Figure S1. Phylogenetic tree of evolutionary relationship of SHANK family proteins. Figure $\mathbf{S 2}$. Homology comparison of zebrafish shank3a and shank3b with human SHANK3. Figure S3. Homology comparison between zebrafish shank3a and shank3b. Figure $\mathbf{S 4}$. shank3b target-mutation in zebrafish via CRISPR-Cas9 system. Figure S5. Examination of maternal or paternal origin effects on the morphological and behavioral phenotypes. Figure S6. Analysis of activity frequency at different activity intensity scales. (PDF $1211 \mathrm{~kb}$ )

Additional file 2: Movie S1. WT zebrafish swimming in the open field. (MP4 $657 \mathrm{~kb}$ )

Additional file 3: Movie S2. shank3b ${ }^{-/-}$zebrafish swimming in repetitive figure "8" pattern. (MP4 $484 \mathrm{~kb}$ )

Additional file 4: Movie S3. shank3b $b^{-1-}$ zebrafish swimming in repetitive big circling pattern. (MP4 $1193 \mathrm{~kb}$ )

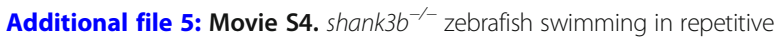
small circling pattern. (MP4 $380 \mathrm{~kb}$ )

Additional file 6: Movie S5. shank $3 b^{-/-}$zebrafish swimming in repetitive walling pattern. (MP4 $1535 \mathrm{~kb}$ )
Additional file 7: Movie S6. Performance of WT zebrafish in the shoaling test. (MPG $2510 \mathrm{~kb}$ )

Additional file 8: Movie S7. Performance of shank36 ${ }^{-1-}$ zebrafish in the shoaling test. (MPG $2022 \mathrm{~kb}$ )

Additional file 9: Movie S8. Performance of WT zebrafish in the social preference test. (MPG $2562 \mathrm{~kb}$ )

Additional file 10: Movie S9. Performance of shank $3 b^{-1-}$ zebrafish in the social preference test. (MPG $1992 \mathrm{~kb}$ )

Additional file 11: Movie S10. Performance of WT zebrafish in the kin recognition and preference test. (MPG $2894 \mathrm{~kb}$ )

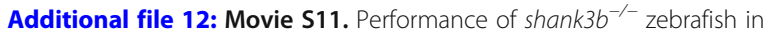
the kin recognition and preference test. (MPG $2044 \mathrm{~kb}$ )

\section{Abbreviations}

ASD: Autism spectrum disorder; dpf: Days post-fertilization; gRNA: GuideRNA; KO: Knockout; mpf: Months post-fertilization; PCR: Polymerase chain reaction; RT-qPCR: Real-time quantitative polymerase chain reaction; SHANK3: SH3 and multiple ankyrin (ANK) repeat domain 3; WT: Wild type

\section{Acknowledgements}

We thank all members of the Division of Child Health Care and Zebrafish Core of Children's Hospital of Fudan University in China; Xiang Yu, Xiao-ming Wang, and Ning Guo for the guidance on the experiments; Xu Wang from Fudan University for providing the $\mathrm{Tg}$ (Huc: RFP) transgenic line; and Dong-yun Li and Samuel Hulbert for critical reading and editing of the manuscript.

\section{Funding}

This study was supported by grants from the National Natural Science Foundation of China (NSFC, no. 81371270) and the National Key Research and Development Program of China (no. 2016YFC1306205) to XX. QL is supported by grants from the NSFC (no. 8127509). YHJ is supported by grants from the National Institute of Health (MH098114, HD087795, and MH104316).

\section{Availability of data and materials}

The datasets generated during the current study are available from the corresponding authors on reasonable request.

\section{Authors' contributions}

The study was conceived by XX. XX, QL and CXL designed the study. CXL performed the experiments. $\mathrm{CXL}, \mathrm{CYL}, \mathrm{CCH}$, and $\mathrm{YW}$ provided homozygous identification assistance. QL and JL provided technical assistance in behavior analyses. CXL wrote the manuscript with comments from all authors. YHJ contributed to the experimental design, data analysis, and manuscript preparation. All authors read and approved the final manuscript.

Ethics approval and consent to participate

The study was approved by the Institutional Review Board of Children's Hospital of Fudan University, China ([2013]024).

\section{Competing interests}

The authors declare that they have no competing interests.

\section{Publisher's Note}

Springer Nature remains neutral with regard to jurisdictional claims in published maps and institutional affiliations.

\section{Author details}

'Division of Child Health Care, Children's Hospital of Fudan University, 399 Wanyuan Road, Shanghai 201102, China. ${ }^{2}$ Center for Translational Medicine, Institute of Pediatrics, Shanghai Key Laboratory of Birth Defect, Children's Hospital of Fudan University, 399 Wanyuan Road, Shanghai 201102, China. ${ }^{3}$ Department of Pediatrics and Neurobiology, Duke University School of Medicine, Durham, NC 27614, USA. 
Received: 26 May 2017 Accepted: 6 March 2018

\section{Published online: 02 April 2018}

\section{References}

1. Monteiro P, Feng G. SHANK proteins: roles at the synapse and in autism spectrum disorder. Nat Rev Neurosci. 2017;18(3):147-57.

2. Jiang $\mathrm{YH}$, Ehlers MD. Modeling autism by SHANK gene mutations in mice. Neuron. 2013;78(1):8-27.

3. Grabrucker AM, Schmeisser MJ, Schoen M, et al. Postsynaptic ProSAP/Shank scaffolds in the cross-hair of synaptopathies. Trends Cell Biol. 2011;21(10): 594-603.

4. Wang X, Bey AL, Katz BM, et al. Altered mGluR5-Homer scaffolds and corticostriatal connectivity in a Shank3 complete knockout model of autism. Nat Commun. 2016;7:11459.

5. Sala C, Vicidomini C, Bigi I, et al. Shank synaptic scaffold proteins: keys to understanding the pathogenesis of autism and other synaptic disorders. J Neurochem. 2015;135(5):849-58.

6. Verpelli C, Dvoretskova E, Vicidomini C, et al. Importance of Shank3 protein in regulating metabotropic glutamate receptor 5 (mGluR5) expression and signaling at synapses. J Biol Chem. 2011;286(40):34839-50.

7. Bonaglia MC, Giorda R, Borgatti $\mathrm{R}$, et al. Disruption of the ProSAP2 gene in a $\mathrm{t}(12 ; 22)(\mathrm{q} 24.1 ; \mathrm{q} 13.3)$ is associated with the 22q13.3 deletion syndrome. Am J Hum Genet. 2001;69(2):261-8

8. Bonaglia MC, Giorda R, Beri S, et al. Molecular mechanisms generating and stabilizing terminal 22q13 deletions in 44 subjects with Phelan/McDermid syndrome. PLoS Genet. 2011;7(7):e1002173.

9. Moessner R, Marshall CR, Sutcliffe JS, et al. Contribution of SHANK3 mutations to autism spectrum disorder. Am J Hum Genet. 2007:81(6):1289-97.

10. Betancur C, Buxbaum JD. SHANK3 haploinsufficiency: a "common" but underdiagnosed highly penetrant monogenic cause of autism spectrum disorders. Mol Autism. 2013;4(1):17.

11. Durand CM, Betancur C, Boeckers TM, et al. Mutations in the gene encoding the synaptic scaffolding protein SHANK3 are associated with autism spectrum disorders. Nat Genet. 2007:39(1):25-7.

12. Mathur P, Guo S. Use of zebrafish as a model to understand mechanisms of addiction and complex neurobehavioral phenotypes. Neurobiol Dis. 2010; 40(1):66-72.

13. Jaramillo TC, Speed HE, Xuan Z, et al. Altered striatal synaptic function and abnormal behaviour in Shank3 Exon4-9 deletion mouse model of autism. Autism Res. 2016;9(3):350-75.

14. Speed HE, Kouser M, Xuan Z, et al. Autism-associated insertion mutation (InsG) of Shank3 exon 21 causes impaired synaptic transmission and behavioral deficits. J Neurosci. 2015:35(26):9648-65.

15. Lee J, Chung C, Ha S, et al. Shank3-mutant mice lacking exon 9 show altered excitation/inhibition balance, enhanced rearing, and spatial memory deficit. Front Cell Neurosci. 2015:9.94.

16. Stewart AM, Nguyen M, Wong K, et al. Developing zebrafish models of autism spectrum disorder (ASD). Prog Neuro-Psychopharmacol Biol Psychiatry. 2014;50:27-36

17. Kalueff AV, Stewart AM, Gerlai R. Zebrafish as an emerging model for studying complex brain disorders. Trends Pharmacol Sci. 2014;35(2):63-75.

18. Liu C, Peng X, Hu C, et al. Developmental profiling of ASD-related shank3 transcripts and their differential regulation by valproic acid in zebrafish. Dev Genes Evol. 2016;226(6):389-400.

19. Kozol RA, Cukier HN, Zou B, et al. Two knockdown models of the autism genes SYNGAP1 and SHANK3 in zebrafish produce similar behavioral phenotypes associated with embryonic disruptions of brain morphogenesis. Hum Mol Genet. 2015;24(14):4006-23.

20. Gauthier J, Champagne N, Lafrenière RG, et al. De novo mutations in the gene encoding the synaptic scaffolding protein SHANK3 in patients ascertained for schizophrenia. Proc Natl Acad Sci. 2010;107(17):7863-8.

21. Mali $P$, Yang $L$, Esvelt KM, et al. RNA-guided human genome engineering via Cas9. Science. 2013:339(6121):823-6.

22. Hwang $W Y, F u, Y$, Reyon D, et al. Efficient genome editing in zebrafish using a CRISPR-Cas system. Nat Biotechnol. 2013:31(3):227-9.

23. Westerfield M. The zebrafish book: a guide for the laboratory use of zebrafish, Brachydanio rerio. Eugene: University of Oregon Press; 1995.

24. Park HC, Kim CH, Bae YK, et al. Analysis of upstream elements in the HuC promoter leads to the establishment of transgenic zebrafish with fluorescent neurons. Dev Biol. 2000;227(2):279-93.
25. Kwan KM, Fujimoto E, Grabher C, et al. The Tol2kit: a multisite gateway-based construction kit for Tol2 transposon transgenesis constructs. Dev Dyn. 2007; 236(11):3088-99.

26. Buske C, Gerlai R. Maturation of shoaling behavior is accompanied by changes in the dopaminergic and serotoninergic systems in zebrafish. Dev Psychobiol. 2012;54(1):28-35.

27. Meshalkina DA, N Kizlyk M, V Kysil E, et al. Zebrafish models of autism spectrum disorder. Exp Neurol. 2017;299(Pt A):207-16.

28. Kalueff AV, Gebhardt M, Stewart AM, et al. Towards a comprehensive catalog of zebrafish behavior 1.0 and beyond. Zebrafish. 2013;10(1):70-86.

29. Miller NY, Gerlai R. Shoaling in zebrafish: what we don't know. Rev Neurosci. 2011;22(1):17-25.

30. Arons $\mathrm{MH}$, Thynne CJ, Grabrucker AM, et al. Autism-associated mutations in ProSAP2/Shank3 impair synaptic transmission and neurexin-neuroliginmediated transsynaptic signaling. J Neurosci. 2012;32(43):14966-78.

31. Kwon SE, Chapman ER. Synaptophysin regulates the kinetics of synaptic vesicle endocytosis in central neurons. Neuron. 2011:70(5):847-54.

32. Sarasua $S M$, Dwivedi $A$, Boccuto $L$, et al. Association between deletion size and important phenotypes expands the genomic region of interest in Phelan-McDermid syndrome (22q13 deletion syndrome). J Med Genet. 2011;48(11):761-6.

33. Soorya L, Kolevzon A, Zweifach J, et al. Prospective investigation of autism and genotype-phenotype correlations in 22q13 deletion syndrome and SHANK3 deficiency. Mol. Autism. 2013:4(1):18.

34. Sarasua SM, Dwivedi A, Boccuto L, et al. 22q13.2q13.32 genomic regions associated with severity of speech delay, developmental delay, and physical features in Phelan-McDermid syndrome. Genet Med. 2014;16(4):318-28.

35. Zhou Y, Kaiser T, Monteiro P, et al. Mice with Shank3 mutations associated with ASD and schizophrenia display both shared and distinct defects. Neuron. 2016;89(1):147-62.

36. Yang M, Bozdagi O, Scattoni ML, et al. Reduced excitatory neurotransmission and mild autism-relevant phenotypes in adolescent Shank3 null mutant mice. Neurosci. 2012:32(19):6525-41.

37. Hoffman EJ, Turner KJ, Fernandez JM, et al. Estrogens suppress a behavioral phenotype in zebrafish mutants of the autism risk gene, CNTNAP2. Neuron. 2016;89(4):725-33.

38. Dadda M, Domenichini A, Piffer $L$, et al. Early differences in epithalamic leftright asymmetry influence lateralization and personality of adult zebrafish. Behav Brain Res. 2010;206(2):208-15.

39. Blaser R, Gerlai R. Behavioral phenotyping in zebrafish: comparison of three behavioral quantification methods. Behav Res Methods. 2006;38(3):456-69.

40. Delaney M, Follet C, Ryan $\mathrm{N}$, et al. Social interaction and distribution of female zebrafish (Danio rerio) in a large aquarium. Biol Bull. 2002;203(2):240-1.

41. D Amico D, Estivill $X$, Terriente J. Switching to zebrafish neurobehavioral models: the obsessive-compulsive disorder paradigm. Eur J Pharmacol. 2015:759:142-50.

42. Peca J, Feliciano C, Ting JT, et al. Shank3 mutant mice display autistic-like behaviours and striatal dysfunction. Nature. 2011:472(7344):437-42.

43. Vicidomini C, Ponzoni L, Lim D, et al. Pharmacological enhancement of mGlu5 receptors rescues behavioral deficits in SHANK3 knock-out mice. Mol Psychiatry. 2017;22(5):784.

44. Hyman SE. A glimmer of light for neuropsychiatric disorders. Nature. 2008; 455(7215):890-3.

45. Tu JC, Xiao B, Naisbitt $\mathrm{S}$, et al. Coupling of mGluR/Homer and PSD-95 complexes by the Shank family of postsynaptic density proteins. Neuron. 1999; 23(3):583-92.

46. Han Q, Kim YH, Wang $X$, et al. SHANK3 deficiency impairs heat hyperalgesia and TRPV1 signaling in primary sensory neurons. Neuron. 2016;92(6):1279-93. 